\title{
Percutaneous Fat Transfer to Treat Knee Osteoarthritis Symptoms: Preliminary Results
}

\author{
Ezio Adriani ${ }^{1}$ Mariagrazia Moio ${ }^{2}$ Berardino Di Paola ${ }^{1}$ Walter Salustri ${ }^{1}$ Andrea Alfieri ${ }^{1}$ Paola Parisi ${ }^{2}$ \\ Marco Ruggiero ${ }^{2}$ Zack Borab ${ }^{2}$ Bruno Carlesimo ${ }^{2}$
}

${ }^{1}$ Department of Orthopedics and Traumatology, Sport Clinique, Rome, Italy

2 Department of Plastic Surgery, Policlinico Umberto I, “La Sapienza” University of Rome, Rome, Italy

Address for correspondence Ezio Adriani, MD, Department of Orthopedics and Traumatology, Sport Clinique, Via Antonio Bertoloni 32, 00197, Rome, Italy (e-mail: ezioadriani@gmail.com).

Joints 2017;5:89-92.

\begin{abstract}
Purpose This study aims to evaluate the safety and efficacy of autologous aspirated and purified fat tissue injected percutaneously into the knee joint for the treatment of symptomatic osteoarthritis (OA).

Methods We reviewed 30 patients, who received an autologous percutaneous fat injection for the treatment of knee OA, from January 2012 to March 2015. Mean patients' age was $63.3 \pm 5.3$ years (range, $50-80$ years). Body mass index was $25.1 \pm 1.7$. Clinical evaluation was based on pain visual analog scale (VAS) and WOMAC score for functional and subjective assessment. We also noted the adverse reactions and the consumption of nonsteroidal anti-inflammatory drugs in the posttreatment period.

Results All patients reported improvements with respect to pain: average VAS was $7.7 \pm 1.2$ at baseline, $5.2 \pm 0.2$ at 1 -month follow-up, and $4.3 \pm 1$ at 3-month follow-

Keywords

- fat tissue

- knee

- osteoarthritis

- injections

- aspirate up. A slight deterioration $(5.0 \pm 1.1)$ was evidenced at 1 year. Total WOMAC score was $89.9 \pm 1.7$ at baseline, $66.3 \pm 1$ at 1 month, $68.6 \pm 1.7$ at 3 months, and $73.2 \pm 1.8$ at 12 months of follow-up.

Conclusion Our preliminary findings suggest that autologous percutaneous fat injections are a valid treatment option for knee OA.

Level of Evidence Level IV, therapeutic case series.
\end{abstract}

\section{Introduction}

The social impact of degenerative diseases of the articular cartilages is steadily increasing due to the continuous increase in the average life expectancy and the rate of active population. ${ }^{1}$ The degeneration of articular cartilage is part of the clinical syndrome of osteoarthritis (OA), representing one of the most common causes of joint pain and disability with related changes in bone morphology in middle-aged and older people. Injured cartilage has a limited ability to heal, and the function of chondrocytes has shown to suffer age-related changes. ${ }^{2}$ Injuries, occupational activities, and obesity appear to be the most common causes of $\mathrm{OA}$ in young and athletic populations. ${ }^{3}$

Biological therapies are gaining increasing popularity in the treatment of degenerative joint diseases, especially in patients with high functional demand. Among the biological treatments, platelet-rich plasma (PRP) is the most investigated. ${ }^{4-6}$ Several studies highlighted that this treatment results in an improvement in knee function, with a reduction of inflammation and consequently a reduction of pain. ${ }^{7-9}$ However, the use of PRP for the treatment of OA is controversial, because none of the studies identifies definitive criteria for patient selection. Although this treatment represent a treatment option when other conservative treatments fail, there is insufficient evidence to recommend the use of PRP. ${ }^{10-12}$

In recent years, the growing attention to the potential applications of regenerative medicine and autologous stem cells has led to the development of new treatments with the aim of helping the regeneration of articular cartilage. Despite the growing interest in this biological approach for cartilage regeneration, knowledge on this topic is still preliminary. ${ }^{13}$ 
In the present study, we evaluated the safety and efficacy of autologous aspirated and purified fat tissue injected percutaneously into the knee joint to treat OA symptoms. We hypothesized that the fat with its content of adiposederived stem cells (ASCs) injected into the joint, might contribute to cartilage healing and meliorate mechanical joint function.

\section{Methods}

\section{Participants}

We retrospectively reviewed 30 patients (12 males and 18 females), who received an autologous percutaneous fat injection for the treatment of knee OA, from January 2012 to March 2015. Mean patients' age was $63.3 \pm 5.3$ years (range, 50-80 years). Body mass index was $25.1 \pm 1.7$. Inclusion criteria were: stable or progressive knee $\mathrm{OA}$ for at least 12 months, no other injective treatments during the last 12 months, no previous knee surgeries, no infections or systemic inflammatory diseases. The diagnosis of OA was made following the American College of Rheumatology criteria and according to the classification of KellgrenLawrence. $^{14,15}$

Patients with narcotic use, non-OA joint pain, systemic conditions, others ongoing or previous injective OA treatments, and age younger than 18 , were excluded. Written informed consent was obtained from all patients before treatment after having explained all the potential risks and benefits of the purposed method and all the possible alternative therapeutic approaches.

\section{Interventions}

Fat was harvested from the abdomen using a 2-mm multiport small-hole cannula and was sheared into finer particles using $60 \mathrm{~mL}$ sterile syringe Luer-Lock with microspheres ( - Fig. 1). After 7 to 10 minutes of infiltration of the donor site with Klein solution (-Fig. 2), $20 \mathrm{~mL}$ of fat was harvested from the abdomen (-Figs. 3 and $\mathbf{4}$ ), and after decantation and separation of the liquid component, $6 \mathrm{ml}$ was injected into each knee ( - Fig. 5) under local anesthesia. Decanted fat was injected percutaneously with $1-\mathrm{mm}$ cannulas. Percutaneous access to the knee articular space was located at the superior-lateral margin of the patella. Patients were asked to avoid sports activities for 7 days after the treatment.

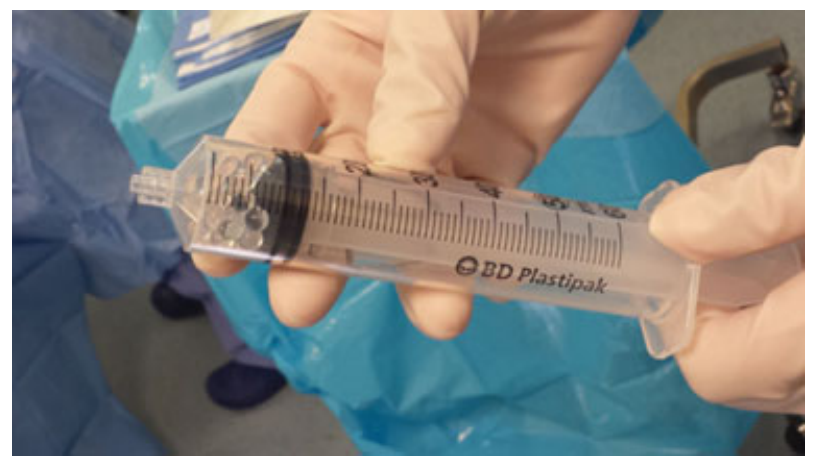

Fig. 1 Sterile Luer-Lock syringe with microspheres.

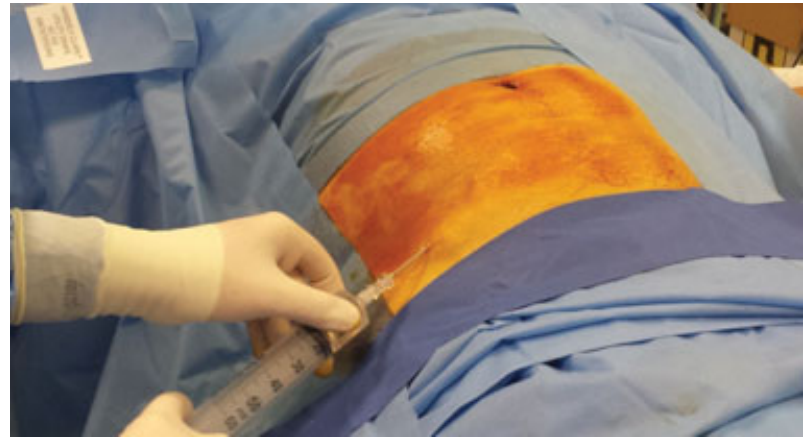

Fig. 2 The donor site is infiltrated with Klein solution.

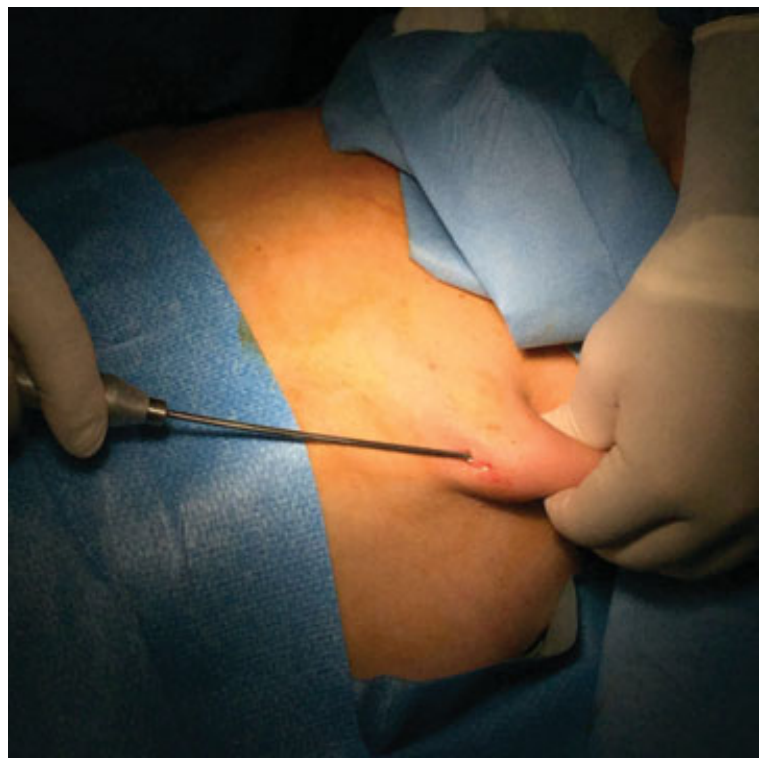

Fig. 3 Fat tissue is taken from the abdomen.

In the postoperative period, an abdominal girdle for 15 days was applied to all patients, to reduce the formation of a local hematoma, while a pressure dressing was applied to the knee for 1 day. All the patients followed a rehabilitation protocol to improve posture and muscle toning.

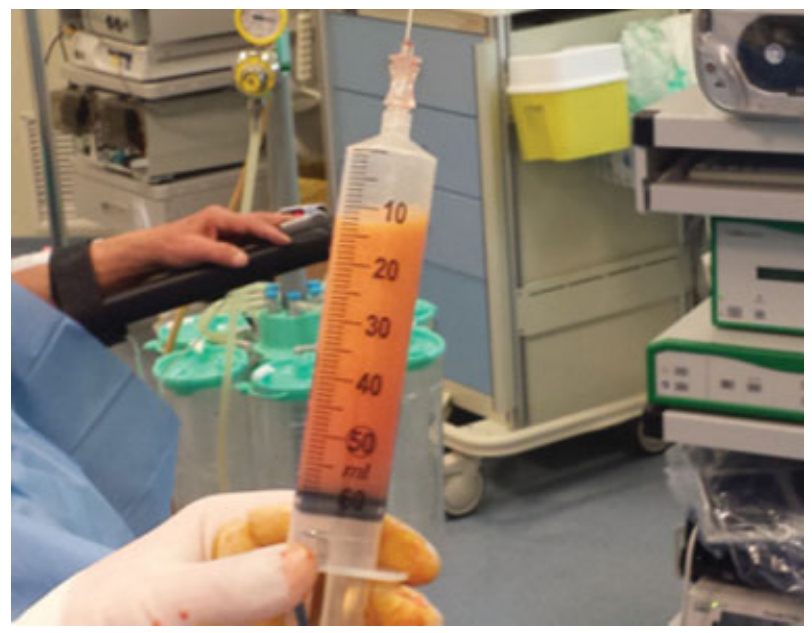

Fig. 4 Fat tissue into the syringe. 


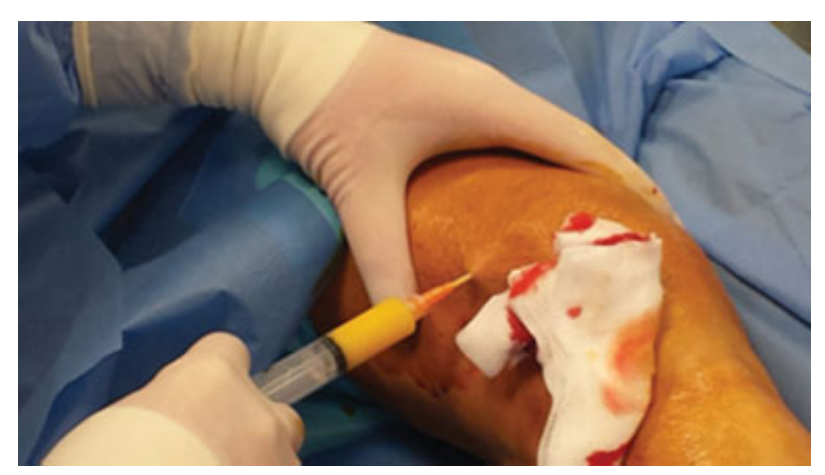

Fig. 5 Fat tissue is injected into the knee.

\section{Outcome Measurements}

The patients were evaluated at baseline and one week and 1, 3, 6, and 12 months after treatment.

A questionnaire was administered to the patients using a rating scale to assess pretreatment and posttreatment pain intensity (at rest, during normal activity, and during exercise), stiffness, the range of motion (ROM), crepitus, and ability to exercise.

Clinical evaluation was based on pain VAS and WOMAC score for functional and subjective assessment. We also noted the adverse reactions and the consumption of nonsteroidal anti-inflammatory drugs in the posttreatment period.

\section{Results}

Patients uniformly expressed satisfaction with outcomes in interviews and all patients reported improvements with respect to pain. Eight patients (26.6\%) achieved complete or near-complete symptoms relief, and 16 patients (53.3\%) achieved resumption of vigorous exercise. Four patients (13.3\%) reported only a slight benefit but were not able to recover a complete ROM. Two patients (6.6\%), who had a grade III OA did not obtain benefits in regard to pain and functional recovery from the treatment.

Average VAS was $7.7 \pm 1.2$ at baseline, $5.2 \pm 0.2$ at 1 month follow-up and $4.3 \pm 1$ at 3-month follow-up. A slight deterioration $(5.0 \pm 1.1)$ was evidenced at 1 year.

Total WOMAC score was $89.9 \pm 1.7$ at baseline, $66.3 \pm 1$ at 1-month follow-up, $68.6 \pm 1.7$ at 3 months, and $73.2 \pm 1.8$ at 12 months of follow-up. Analysis of the WOMAC subscores confirmed evidence of a relative deterioration of pain over time (17.8 \pm 0.3 at baseline, $11.7 \pm 0.3$ at 1 month, and $14.1 \pm$ at 12 months), which is also accompanied by a deterioration of joint stiffness ( $7.1 \pm 0.2$ at baseline, $3.4 \pm 0.3$ at 1 month, and $4.8 \pm 0.2$ at 12 months) and function ( $63.7 \pm 1.3$ at baseline, $51.2 \pm 1.4$ at 1 month, and $55.8 \pm 1.3$ at 12 months).

Adverse events were recognized as related to harvesting and grafting procedures. In regard to harvesting complications, one patient developed pain in the abdominal region with important hematoma; other two patients developed a hematoma of the abdominal region but it was less important, and consequently, the pain did not affect the initial shooting.
In regard to grafting complications, two patients developed joint swelling that required an aspiration resulting in resolution of symptoms; three other patients developed mild swelling that resolved during rehabilitation. We found neither infections nor neurovascular complications.

We noted a reduction of analgesics consumption in the postoperative period.

\section{Discussion}

Degenerative joint diseases represent a major and growing cause of disability and health care resource consumption. According to the Johnston County Osteoarthritis Project, a long-term study from the University of North Carolina the lifetime risk of developing OA of the knee is approximately $46 \%$, and the lifetime risk of developing OA of the hip is $25 \%{ }^{16}$

Although the most common cause of osteoarthritis of the knee is age, joint injuries can accelerate chondrocyte senescence, and this acceleration plays a role in the joint degeneration responsible for posttraumatic osteoarthritis. ${ }^{17}$ All components of the cartilage play different roles in the stabilization and protection of the joint. Alterations in the structure of the articular cartilage lead to injury and joint degeneration. ${ }^{18}$

Knee OA treatment options include nonpharmacological treatments such as patient education and self-management strategies, advising weight loss and strengthening programs. Oral analgesics and anti-inflammatories are pharmacological options that are commonly used and can be helpful for managing knee OA in the short-term but are less effective for long-term management. Intra-articular injections are also employed to manage knee $\mathrm{OA}$ ranging from corticosteroids to hyaluronic acid to more recently PRP and even stem cells while several other injection therapies are presently being studied. ${ }^{9}$ Autologous platelets are expected to release chondrogenic growth factors following the activation of clotting pathways and several studies support the efficacy of PRP for OA. ${ }^{19-21}$ Injection of ASCs following arthroscopic debridement or PRP with hyaluronic acid yielded improved clinical outcomes using the WOMAC index, Lysholm, and VAS pain score 3 months postoperatively, along with increased cartilage thickness on magnetic resonance imaging. ${ }^{22}$

The development of percutaneous interventions that potentially enhance regenerative processes has improved the chances for nonsurgical treatments that produce durable improvement in pain and function. ${ }^{13}$ Current use of adipose tissue as an injectable includes augmenting tissue volume and correcting contour irregularities, but recent applications promise to capture fat's immunomodulatory and regenerative capacity. Adipose tissue is an important source of mesenchymal stem cells (MSCs). Compared with bone marrow-derived MSCs, ASCs from lipoaspirates can be harvested using a less-invasive procedure and in larger amounts. Moreover, they can commit toward the chondrogenic, osteogenic, adipogenic, myogenic, and neurogenic lineages. ${ }^{23}$

Our study was conducted using an intra-articular percutaneous injection of processed and decanted subcutaneous fat, a simple and inexpensive procedure, to treat a series of 
patients displaying degenerative joint ailments. Whole fat injection potentially captures elements of several regenerative strategies. In contrast to prior ASCs therapies, fat is not processed to separate MSCs, and therefore potentially supportive biomechanical functions of the fat tissue are retained in addition to ASCs. Adipose cells could, in fact, enhance the biological function of Hoffa's infrapatellar fat pad as an anatomical spacer between the articular surfaces. Different studies showed that ASCs obtained from subcutaneous adipose tissue could be a viable approach for treating OA, but cells manipulation to extract them from fat is considered an advanced therapy, thus regulated by the Committee for Advanced Therapies of the European Medicines Agency with specific rules concerning the authorization, supervision, and pharmacovigilance of advanced therapy medicinal products. On the other hand, intraoperative use of autologous and nonmanipulated fat could provide a source of ASCs easily accessible and with potential regenerative and biomechanical benefits in the treatment of knee OA.

Preclinical and clinical studies on the use of autologous MSCs for chondrogenic repair have focused on preparations in which MSCs are enriched and expanded, with the assumption that quantity of delivered MSC is critical. This assumption, however, remains untested and recent studies suggest that the chondrogenic action of MSC may depend more on trophic functions, including the secretion of angiogenic factors, such as vascular endothelial growth factor, than on the chondrocyte differentiation and structural incorporation of these cells. ${ }^{24,25}$

In conclusion, this study was motivated by the desire to develop simple, rapid, inexpensive options with low morbidity for use in outpatient settings. Our preliminary findings suggest that whole autologous percutaneous fat injection merits investigation as a possible alternative treatment for knee OA.

\section{References}

1 Curl WW, Krome J, Gordon ES, Rushing J, Smith BP, Poehling GG. Cartilage injuries: a review of 31,516 knee arthroscopies. Arthroscopy 1997;13(04):456-460

2 Buckwalter JA, Mankin HJ. Articular cartilage: degeneration and osteoarthritis, repair, regeneration, and transplantation. Instr Course Lect 1998;47:487-504

3 Amoako AO, Pujalte GGA. Osteoarthritis in young, active, and athletic individuals. Clin Med Insights Arthritis Musculoskelet Disord 2014;7:27-32

4 Mazzucco L, Balbo V, Cattana E, Guaschino R, Borzini P. Not every PRP-gel is born equal. Evaluation of growth factor availability for tissues through four PRP-gel preparations: Fibrinet, RegenPRPKit, Plateltex and one manual procedure. Vox Sang 2009;97(02): 110-118

5 Molloy T, Wang Y, Murrell G. The roles of growth factors in tendon and ligament healing. Sports Med 2003;33(05):381-394

6 Staudenmaier R, Froelich K, Birner M, et al. Optimization of platelet isolation and extraction of autogenous TGF-beta in cartilage tissue engineering. Artif Cells Blood Substit Immobil Biotechnol 2009;37(06):265-272

7 Paterson KL, Nicholls M, Bennell KL, Bates D. Intra-articular injection of photo-activated platelet-rich plasma in patients with knee osteoarthritis: a double-blind, randomized controlled pilot study. BMC Musculoskelet Disord 2016;17(01):67. Doi: 10.1186/s12891-016-0920-3

8 Kavadar G, Demircioglu DT, Celik MY, Emre TY. Effectiveness of platelet-rich plasma in the treatment of moderate knee osteoarthritis: a randomized prospective study. J Phys Ther Sci 2015; 27(12):3863-3867

9 Smith PA. Intra-articular autologous conditioned plasma injections provide safe and efficacious treatment for knee osteoarthritis: An FDA- sanctioned, randomized, double-blind, placebocontrolled clinical trial. Am J Sports Med 2016;44(04):884-891

10 Moraes VY, Lenza M, Tamaoki MJ, Faloppa F, Belloti JC. Platelet-rich therapies for musculoskeletal soft tissue injuries. Cochrane Database Syst Rev 2014;4(04):CD010071. Doi: 10.1002/ 14651858.CD010071. pub2

11 Grambart ST. Sports medicine and platelet-rich plasma: nonsurgical therapy. Clin Podiatr Med Surg 2015;32(01):99-107

12 Duymus TM, Mutlu S, Dernek B, Komur B, Aydogmus S, Kesiktas FN. Choice of intra-articular injection in treatment of knee osteoarthritis: platelet-rich plasma, hyaluronic acid or ozone options. Knee Surg Sports Traumatol Arthrosc 2017;25(02):485-492

13 DeChellis DM, Cortazzo MH. Regenerative medicine in the field of pain medicine: Prolotherapy, platelet-rich plasma therapy, and stem cell therapy-Theory and evidence. Techniques in Regional Anesthesia and Pain Management 2011;15(02):74-80

14 Misso ML, Pitt VJ, Jones KM, Barnes HN, Piterman L, Green SE. Quality and consistency of clinical practice guidelines for diagnosis and management of osteoarthritis of the hip and knee: a descriptive overview of published guidelines. Med J Aust 2008; 189(07):394-399

15 Kijowski R, Blankenbaker D, Stanton P, Fine J, De Smet A. Arthroscopic validation of radiographic grading scales of osteoarthritis of the tibiofemoral joint. Am J Roentgenol 2006;187 (03):794-799

16 Nelson AE, Renner JB, Schwartz TA, Kraus VB, Helmick CG, Jordan JM. Differences in multijoint radiographic osteoarthritis phenotypes among African Americans and Caucasians: the Johnston County Osteoarthritis project. Arthritis Rheum 2011;63(12): 3843-3852

17 Martin JA, Brown T, Heiner A, Buckwalter JA. Post-traumatic osteoarthritis: the role of accelerated chondrocyte senescence. Biorheology 2004;41(3-4):479-491

18 Levangie PK, Norkin CC. Joint Structure and Function: A Comprehensive Analysis. 3rd ed. Philadelphia: FA Davis; 2005:80

19 Kon E, Buda R, Filardo G, et al. Platelet-rich plasma: intra-articular knee injections produced favorable results on degenerative cartilage lesions. Knee Surg Sports Traumatol Arthrosc 2010;18(04): 472-479

20 Fibel KH, Hillstrom HJ, Halpern BC. State-of-the-art management of knee osteoarthritis. World J Clin Cases 2015;3(02):89-101

21 Sánchez M, Anitua E, Azofra J, Aguirre JJ, Andia I. Intra-articular injection of an autologous preparation rich in growth factors for the treatment of knee OA: a retrospective cohort study. Clin Exp Rheumatol 2008;26(05):910-913

22 Pak J. Regeneration of human bones in hip osteonecrosis and human cartilage in knee osteoarthritis with autologous adiposetissue-derived stem cells: a case series. J Med Case Reports 2011; 5:296-303

23 Izadpanah R, Trygg C, Patel B, et al. Biologic properties of mesenchymal stem cells derived from bone marrow and adipose tissue. J Cell Biochem 2006;99(05):1285-1297

24 Ankrum J, Karp JM. Mesenchymal stem cell therapy: Two steps forward, one step back. Trends Mol Med 2010;16(05):203-209

25 Tögel F, Weiss K, Yang Y, Hu Z, Zhang P, Westenfelder C. Vasculotropic, paracrine actions of infused mesenchymal stem cells are important to the recovery from acute kidney injury. Am J Physiol Renal Physiol 2007;292(05):F1626-F1635 